\title{
Explicit Evaluation of Complexity in Construction and Real Estate Management
}

\author{
Wolfgang Eber
}

Technische Universität München, Lehrstuhl für Bauprozessmanagement, München, Germany, Ibi@bv.tum.de

\begin{abstract}
Complexity has become a major keyword when it comes to organization issues in Construction and Real Estate Management. Expressed via many different theoretical definitions, complexity in terms of management represents the part of organization which is simply not manageable. Therefore, reduction of complexity by separating a system into independent well-defined and well-controlled subsystems becomes a major task. However, since construction and real estate projects are becoming larger, encompassing higher volumes as well as higher numbers of participants and are nonetheless subjected to strongly limited time-frames and budgets on tight markets, efficient organization developed into the crucial issue to stand a competition successfully.
\end{abstract}

On this background, engineering naturally focuses on saving costly resources, where the explicit value of a single measure can easily be derived from the cost of the resource and the duration of the therewith reduced time floats. This leads to well-known concepts, e.g. just-in-time-delivery, where a system is optimized with respect to physical resources as well as virtual resources like storage space or reserve time. However, as this strategy clearly saves explicit local resources, concurrently the coupling of processes via the required availability of physical and virtual resources, ranging from pre-products to decisions, plans and responsibilities, is strongly increased and, thus, complexity is reintroduced to a significant degree.

This paper proposes an approach on the basis of Systems Theory providing an explicit measure to evaluate the increase of complexity in relation to possibly saved resources. Since cost of complexity are not given a priori but result from possible deviations, this article investigates the propagation of virtual uncertainties of real and abstract pre-products through a network of given complexity. On this basis, some general rules are derived allowing to maintain the balance between saving resources and the therewith increasing cost of the consequently rising complexity.

(c) 2020 The Authors. Published by Budapest University of Technology and Economics \& Diamond Congress Ltd Peer-review under responsibility of the Scientific Committee of the Creative Construction Conference 2020.

Keywords: complexity, systems theory, lean management, construction management, real estate management

\section{Introduction}

Long since, construction management suffered from the very specific task to get the production of a unique product organised [1, 2], in particular within a heavily specialised context [3]. Based on the definition of general processes, the uniqueness of products no longer poses a problem as the production as well as the planning processes become standardized [4] and the uniqueness is reduced to concatenating elements in a specific way [5]. Then, only a limited number of parameters describes the standardized processes and the task is changed to precisely determining these in advance. The processes themselves are predetermined (see e.g. [6]) and equipped with superordinate control-mechanisms ensuring the required results within the given margins of quality, cost and time [7]. 
So far, the overall production is formed by a system of interacting elements/objects [8] which can be described by the Theory of Systems $[9,10,11]$ accordingly. Elements may be given as physical modules as e.g. in Building Information Models (BIM) [6], however, in this context they are exclusively understood as processes. Depending on type, direction and strength of coupling these objects, such systems tend to develop dynamical behaviour [12,13], which needs to be understood and taken into account $[14,11,15]$.

A well-known side-effect of the standardisation of processes is the new focus on the concatenation parameters, drifting away from the actual content of the processes as is intended. However, as a consequence, the production processes, in particular including the respective controlling structures, are expected to fulfil their task perfectly well [16].

On this background, planning is reduced to the - nonetheless demanding - task, to define the complete set of parameters coupling the numerous processes which in the end lead to the final product. So far, project management focuses firstly on the separation of the overall task into a number of processes in a wellstructured way and secondly on reconnecting them into an output-oriented production system $[17,18,19$, 20].

The very engineering-like approach to optimize this with respect to the consumption of resources focuses on the total set of processes, in particular avoiding any idle running as the major wastage [21, 22, 23]. In order to do so under the pressure of tight schedules and low margins, pains are taken to couple processes as closely as possible, thus minimizing time floats and tolerance regarding perfection of pre-products [24].

Since tight and numerous coupling of a set of processes is measured in terms of complexity or connectivity $[25,26,27,20]$, this inevitably leads to an increase of complexity of the respective projects $[28,29,30]$. In contrast, it is commonly well-known that reduction of complexity is an important general goal [31, 32, 33], however, there is no quantitative description of measures and consequences available. As a significant number of current projects, in particular in construction management, teach, despite very careful planning and investing high engineering skills, these tend to fail their ambitious goals, probably due to the introduction of an unmanageable degree of complexity [34].

In general, there are two major issues to be considered in this situation. First, investing resources in controlling mechanisms allows for safely achieving given goals, however, only within well-determined limits which directly depend on the quantity of available controlling resources. Therefore, still some well-defined degree of indeterminacy remains and needs to be taken into account [35]. On this background, secondly, as the qualitative understanding of principal fuzziness grew, rules of "Lean Management" are discussed and investigated $[21,22,23]$. One of the there mentioned aspects, in very short, is to focus on the final production processes since these are generating most of the value and they are most likely to suffer most from any deviations. This is in contrast to classical hierarchical approaches which pay most attention to the processes close to the start of causal chains in order not to bring in any deviations at all.

In this paper we propose a formal model of understanding of the major balance of saving resources by ensuring very safe intermediate products and the inherent cost of complexity introduced by the therefrom resulting tight coupling of processes. On the very fundamental basis of systems theory, finally some rules to avoid unpredictable results are developed and recommended.

\section{Complex network situation}

The approach presented here is based on the structure of a general network of processes, where most processes are dependent on the outcome of some other processes. This structure is principally modelled as a graph comprising predecessor nodes and successor nodes connected by directed edges indicating direct dependencies. Following the principle of a graph-theoretical network plan can be safely assumed that the graph is loop-less and holds exactly one source and one sink node $[17,4,18,2]$. Thus, a strictly causal sequence can be determined and formulated as a graph-theoretical rank [16]. Furthermore, in accordance to the definition of activities, linear production as well as linear consumption of temporal and consumable resources is given [5]. 
Understood as activities, these processes make use of resources, partly assigned solely to the particular process, partly shared between several processes. Under all circumstances, the activity-nodes are coupled either by the availability of shared resources or by dependencies introduced by the requirement of preproducts.

All activities are assumed to be well-defined under the responsibility of a perfectly hierarchical approach according to a graph-theoretical tree-structure and, thus, the required methods, results and resources are unambiguously given. On this basis, sensibly, all processes are equipped with appropriate control-loops making sure that possible deviations remain within a given corridor. Nevertheless, even with very tight controlling deviations will occur but remain limited [15, 16].

Finally needs to be preconditioned and accepted, that since deviations of the output of a process lead to deviating input values of the succeeding nodes they therefore travel through the network.

All types of interdependency can easily be expressed as directed edges, so that only a ranked network plan needs to be considered as the fundamental structure to be investigated.

\subsection{Local deviation of a single process/activity}

The result of a process can be formulated as the value of a single variable, which corresponds to some degree to the target value. In this context a varying duration $D$ corresponds directly to a varying result since the linearity of an activity allows achieving the target value consuming respectively more or less time. Therefore, all kinds of deviation of production can as well be expressed as undertime or overtime. In order to maintain linearity and, due to no better information, the given deviation of $D \rightarrow D \pm \tau / 2$ at max is assumed following a constant distribution: $P(\tau) d t=\tau^{-1} d t$

\subsection{Interaction of processes (Activities)}

In general, networks with no invisible deeper structure are described by some well-known parameters [10, $30,25]$ : The impact $\zeta$ counts the average number of direct successors to any other node, interoperability $\xi$ describes the average number of direct predecessors. Complexity $\alpha$ of a network is furthermore formulated as the information [25] within a step proceeding from a node to an average successor with respect to the maximum information: $\alpha=\ln (\xi+1) / \ln N$

For the present situation, the number $\xi$ of direct predecessors to a node defines the precondition of the successor $\mathrm{S}$ to start, therefore we assume the activity $\mathrm{S}$ to require all $\xi$ activities to be completed and finished to the predefined outcome and time.

As pointed out in [30] and based on a constant distribution, the relative loss to a $\xi=1$ situation is $\hat{\tau}_{A D D}(\xi)=(\xi-1) /(\xi+1)$ in units of $\tau / 2$, which reflects the probability of $\xi$ activities to be all finished to perfection.

\subsection{Local interaction of deviating processes/activities, separability and coupling}

In order to avoid transferring any deviation resulting from a preceding activity the consequent process needs to allow for a reserve time which at least equals the maximum overtime $\tau / 2$, i.e. tightness of coupling is set allowing for full reserves $\tau / 2$. This situation, where each activity runs on its own, is addressed as "completely separated".

The resources required for full separability are under these circumstances given by $R_{S}=\tau / 2 \cdot \xi \cdot \eta$, where $\tau / 2$ is the possible deviation, $\xi$ the number of required activities and $\eta$ the required resources per time.

For this approach we generally assume an average value $\eta$ representing cost per time which is assigned to the resources needed for production but as well stands for the generation of value on the basis of these resources. 
In contrast to the fully separated situation, a complete transfer of deviations, i.e. allowing for no reserves $\tau / 2$ per preceding activity, the succeeding process $S$ is understood as "closely coupled". The therewith saved resources would be $R_{S}=\tau / 2 \cdot \xi \cdot \eta$, however, the taken risk develops from the requirement of consuming adequate resources according to the probability of at least one pre-process to fail to some degree:

$$
R_{C}=\frac{\tau}{2} \cdot \eta^{\xi} \frac{\xi-1}{\xi+1}
$$

where $\tau / 2$ is the possible deviation, $\eta^{\xi}$ the resources per time to the power of $\xi$ due to the increase of value with joining processes, and $(\xi-1) /(\xi+1)$ the probability of one out of $\xi$ pre-processes to fail. The background of the approach $\eta^{\xi}$ as generation of value is the understanding of the value of a subsequent process being formed by predecessors as factors. Therefore, the generation of value of the predecessors are to be multiplied yielding the value of the successor.

\subsection{Local interaction of deviating processes (Activities) - Balance}

The balance of both the extreme situations compares the resources saved by tight coupling to the possibly taken risk earned by introducing this degree of complexity

$$
R_{\Delta}=-R_{S}+R_{C}=-\frac{\tau}{2} \cdot \xi \cdot \eta+\frac{\tau}{2} \cdot \eta^{\xi} \frac{\xi-1}{\xi+1}=\frac{\tau}{2} \eta\left(\eta^{\xi-1} \frac{\xi-1}{\xi+1}-\xi\right)=\frac{\tau}{2} \Delta_{l o c} \stackrel{!}{=} 0
$$

As long as $\tau=0$, i.e. no deviation is possible, meaning only perfectly stable and safe results are presented to the next rank, $R_{\Delta}=0$ and no advantages can be taken from avoiding complexity.

\section{Connectivity of network development of cost}

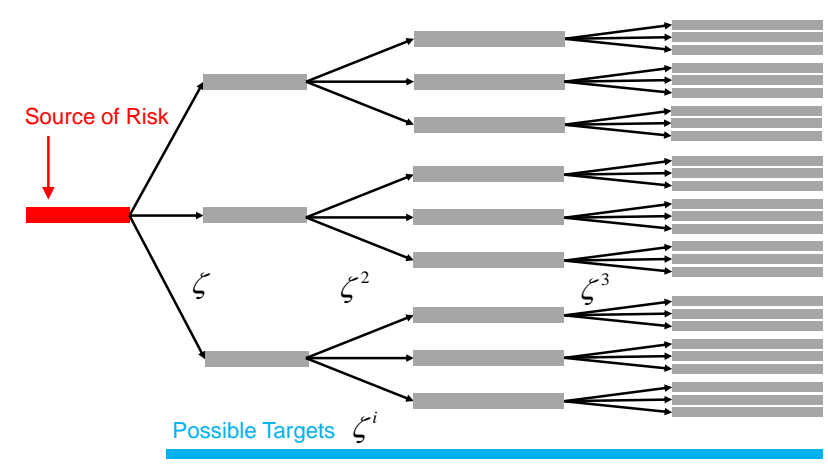

Fig.1. Development of a risk through a network tree.

Any deviation as a consequence of failed completion somewhere within the network is assumed to be carried throughout the network until the causal chains are terminated. The given interoperability $\zeta=\xi=v$ describes the number of affected consecutive processes per level. In this consideration, losses by delayed processes are accumulated for all affected processes, since any deviation from a pre-set schedule requires the presence of the scheduled resources beyond the scheduled time. So far, the structure of a graphtheoretical tree is assumed. Branches may in reality very well be reunited, forming an incomplete tree. However, recombining might mean recombining the same problem twice. Thus, the complete treestructure needs to be taken into account. Therefore, the number of affected processes per level is $\xi^{i}$ where the number of levels $i$ is corresponding to the cardinality $N$ of the order of the maximum rank $\Gamma$, i.e. the length of causal chains. Therewith, any deviation leads to a number of $z$ affected processes.

$$
z=1+\xi+\xi^{2}+\xi^{3}+\ldots=\sum_{i=0}^{\Gamma} \xi^{i}=\frac{\xi^{\Gamma+1}-1}{\xi-1}
$$




\subsection{Connectivity of network development of cost - Balance}

In this set-up, additional loss of resources of all the affected processes needs to be considered cumulatively when balancing saving resources by increasing tightness of linkage.

$$
\Delta_{n e t}=\eta^{\xi-1} \frac{\xi-1}{\xi+1} \cdot \frac{\xi^{\Gamma+1}-1}{\xi-1}-\xi=\frac{\xi-1}{\xi+1} \cdot \frac{\xi^{\Gamma+1}-1}{\xi-1}-\xi
$$

where the generation of value remains unconsidered $(\eta=1)$.

A network never consists of only tight interactions or links with sufficient separation indicating complete decoupling. The degree of coupling $\chi \in[0 . .1]$ reflects the percentage of links in a network being closely coupled. Since consumption of resources as well as the generation of value is linear with all low-level processes (activities), this is not necessarily the number of links but can as well describe the degree of separation for each single link $j$ ranging from close coupling $\left(\chi_{j}=1\right)$ to complete separation $\left(\chi_{j}=0\right)$. Then, $\chi$ also holds the average degree of coupling over the complete network and stands for the probability of a risk to meet a tight interaction transferring all the deviation to the next rank.

This in particular means that only a share of $\chi \xi$ interactions are transferring the deviation and, secondly, only a share of $\chi \Gamma$ levels contribute to the transfer. Hence, we have

$$
\Delta_{n e t}=\frac{\xi-1}{\xi+1} \cdot \frac{(\xi \chi)^{\Gamma \chi+1}-1}{\xi \chi-1}-\xi
$$

This approach allows for some first observations:

The local saving potential (last term) is linear with $\xi$.

The local risk (pre-factor of first term) also runs linear with $\xi$.

The network impact (main factor of first term) is dominating.

From this we derive the principal criteria of balance considering the network. The non-constant term would certainly positively escalate with

$\xi \chi>1$ implicating an escalating numerator as long as causal chains are long enough to provide high powers,

$\xi \chi=1$ leading to a vanishing denominator and, thus, also escalating behaviour,

$\Gamma \chi>1$ where a large size of a network segment would cause over-linear behaviour

Therefore, to keep losses from escalating, we establish some principal limits to maintain balance:

Keeping the longitudinal coupling low, i.e. $\Gamma \chi \rightarrow 1$ leads to $\chi \ll 1 / \Gamma$. therewith coupling is kept to a single level, which is not applicable.

Keeping the lateral coupling low, i.e. $\xi \chi \rightarrow 1$ leads to $\chi \ll 1 / \xi$, which may be used as a helpful criterion.

\subsection{Connectivity of network development of cost - Balance characteristics}

The explicit characteristics in close proximity to the equilibrium state can easily be derived. Holding the criterion $\xi \chi \rightarrow 1$, the development along the network is just linear infinite allowing to integrate the longitudinal coupling to the lateral coupling criterion 


$$
\frac{(\xi \chi)^{\Gamma \chi+1}-1}{\xi \chi-1}=\sum_{i=0}^{\Gamma \chi}(\xi \chi=1)^{i}=\sum_{i=0}^{\Gamma \chi} 1=\Gamma \chi
$$

This allows for further estimations to ignore the variation of the exponent $\Gamma \chi+1$ and introduce the symbolic length of effective causal chains $\Gamma=\Gamma \chi+1$. Exemplarily for $\Gamma=5$, we obtain the cost $\Delta_{\text {net }}$ in terms of saved resources or inherited risk in dependence of the connectivity $\xi$ of the network and the degree of coupling $\chi$.

Cost $\Delta_{\text {net }}$ are generally rising from negative values reaching an equilibrium state and then start escalating with increasing degree of coupling $\chi$. The gradient is negligible for $\xi=1$ as expected for a single linear chain but shows significant increase with higher $\xi>1$. The yellow line indicates equilibrium, i.e. $\Delta_{n e t}(\chi, \xi)=0$. Figure 11 indicates the balance state for a set of different set of $\Gamma$.

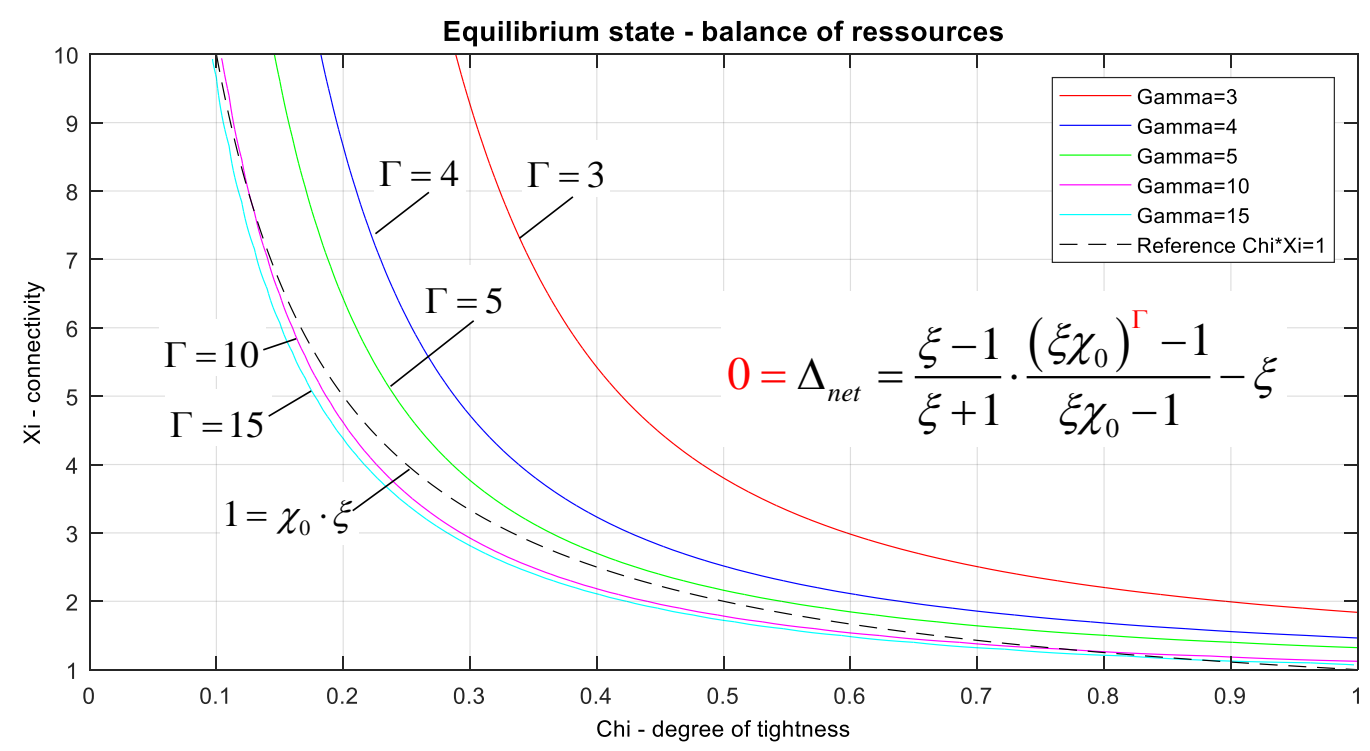

Fig.2. State of equilibrium depending on connectivity $\xi$ and degree of coupling $\chi$.

For comparison, the criterion $\widetilde{\chi_{0}} \cdot \xi=1$ is plotted as well (dashed line). For $\xi=1$ (linear chain) all deviations are averaging and therefore have no accumulating consequences. With rising connectivity, the available range of coupling is significantly reduced. Very short causal sequences allow for not much restrictions. However, clearly can be seen that for $\Gamma>5$ equilibrium can in fact be approximated by the rough rule of thumb $\chi_{0} \cdot \xi \approx 1$, indicating for example that a connectivity of e.g. $\xi=10$ restricts the sensible degree of coupling to $\chi_{0} \approx 10 \%$.

\section{Conclusion}

The simple model presented here certainly does not cover all possible details of interaction and network characteristics. In particular consumption of resources and utilization of pre-products is strongly simplified here. However, as long as the linear dependency character of processes can be accepted - as is the definition of low-level activities - the principal outcome provides some inevitable reasoning.

The fundamental parameter of the network would be its complexity $\alpha=\ln (\xi+1) / \ln N$, respectively in other words $\xi=N^{\alpha}-1=K / N$. A completely non-complex system is characterized by $\alpha=0$ resp. $\xi=0$ indicating absolutely separated processes. As soon as interaction comes into play to some degree $0 \leq \chi \leq 1$, any possible deviation $\tau \geq 0$ is carried and multiplied through any kind of network. Despite a large number of unknown effects, a limit can be provided, up to which coupling, i.e. $\chi \geq 0$, leads to saving 
resources. Exceeding this limit quickly leads to a significant rise of cost due to the given complexity and should be avoided as a primary rule.

This limit is given by roughly a unity square with side-length of one, where the lateral dimension is $\chi \xi=1$ while the longitudinal dimension is $\chi \Gamma=1$. As long as the network-parameters in combination with the degree of coupling are well located within this square, a system may be considered stable and safe. Approaching this limit clearly provides the optimal situation, while exceeding leads inevitably to instable settings. Due to considering an equilibrium situation, the explicit value of the local uncertainty $\tau$ plays no role within this context as long as $\tau>0$. In the end, the gradient at balance runs proportional to $\tau>0$, so that any significant uncertainty allows this rule to remain applicable.

Practically speaking, any network, be it a network plan in construction or real estate management as well as a train schedule, is defined by the length of causal chains $\Gamma$ and the connectivity $\xi$. Since fuzziness cannot be excluded generally, the degree of tight coupling $\chi$ needs to be kept beyond $1 / \Gamma$ as well as beyond $1 / \xi$, say a network with given connectivity of e.g. $\xi \approx 1$ allows for a maximum coupling of $10 \%$ in order to maintain a safe and stable system.

\section{References}

[1] Picot A.; Dietl H.; Franck E. (2008), Organisation - Eine ökonomische Perspektive, 5. rev. ed., ISBN 978-379-102371-7, SchäfferPoeschel, Stuttgart

[2] Schulte-Zurhausen, M. (2002), Organisation, 3rd ed., Verlag Franz Vahlen, München.

[3] Coase, R (1937) The Nature of the Firm Economica, New Series. vol 416 https://doi.org/10.1111/j.1468-0335.1937.tb00002.x

[4] Kerzner, H. (2003). Project Management: A Systems Approach to Planning, Scheduling, and Controlling (8th ed.), ISBN 0884222245414, Wiley, Berlin.

[5] Eber, W. (2019) Principles Of Project Management, Lecture Notes, Chair Of Construction Management, TUM Department of Civil, Geo and Environmental Engineering, Technical University of Munich.

[6] Borrmann, A.; König, M.; Koch, C.; Beetz, J. (2015) Building Information Modeling, Technologische Grundlagen und industrielle Praxis, Springer Vieweg Verlag, Springer Fachmedien Wiesbaden, https://doi.org/10.1007/978-3-658-05606-3

[7] Eber, W. (2018) Das „Building Information Model“ (BIM), eine kritische Würdigung aus Sicht der Systemtheorie Proc. 4. BIM Symposium des BIM Clusters Rheinland-Pfalz, Techn. Universität Kaiserslautern, Baubetrieb und Bauwirtschaft, October 16th 2018, Kaiserslautern

[8] Booch G et al. (2007) Objectorientierted Analysis and Design, 3rd ed. ISBN-13: 978-8131722879, Addison-Wesley, Bonn, 2007

[9] Bertalaffny, L. (1969) General Systems Theory, ISBN 780807604533 George Braziller Inc. New York 1969, P 54 ff.

[10] Eber, W.; Zimmermann, J. (2018), Evaluating and Retrieving Parameters for Optimizing Organizational Structures in Real Estate and Construction Management, Periodica Polytechnica Architecture, Budapest University of Technology and Economics, Budapest, Hungary, 2018. 49 pp 155-164, https://doi.org/10.3311/PPar.12709

[11] Luhmann, N. (2001) Soziale Systeme. Grundriß einer allgemeinen Theorie. ISBN 3-518-28266-2, Frankfurt am Main 1984. (ISBN 3518-28266-2)

[12] Wiener, N. (1992), Kybernetik, ISBN 978-3430196529, Econ Verlag, Düsseldorf, Wien, New York, Moskau.

[13] Zimmermann, J.; Eber, W. (2014) Mathematical Background of Key Performance Indicators for Organizational Structures in Construction and Real Estate Management, Procedia Engineering, Volume 85, 2014, Pages 571-580, https://doi.org/10.1016/j.proeng.2014.10.585

[14] Haken, H. (1983), Synergetik, ISBN 978-3540110507, Springer Verlag, Berlin, Heidelberg, New York, Tokyo.

[15] Malik, F. (2003), Systemisches Management, Evolution, Selbstorganisation, 4th ed., ISBN 3834964409, Haupt Verlag, Bern

[16] Zimmermann, J., Eber, W. (2012), Development of heuristic indicators of stability of complex projects in Real Estate Management, 7th International Scientific Conference, Vilnius, Lithuania, Business and Management 2012, , selected papers, Volume I and II, Vilnius, Lithuania., https://doi.org/10.1007/978-3-322-81523-1

[17] Deutsches Institut für Normung e.V, DIN 69901-5 (2009): Projektmanagement - Projektmanagementsysteme, 9th ed; Beuth, Berlin

[18] Schelle, Ottmann, Pfeiffer (2005), Project Manager, GPM Deutsche Gesellschaft für Projektmanagement, ISBN 9783800637362 , Nürnberg

[19] Zimmermann, J., Eber, W. (2017), Criteria on the Value of Expert's Opinions for Analyzing Complex Structures in Construction and Real Estate Management, Creative Construction Conference 2017, CCC 2017, 19-22 June 2017, Primosten, Croatia, Procedia Engineering, Volume 196, 2017, Pages 335-342, https://doi.org/10.1016/j.proeng.2017.07.208

[20] Zimmermann, J.; Eber, W. (2018) Optimizing Organizational Structures in Real Estate and Construction Management Proc. Creative Construction Conference 2018, CCC 2018, June 30th Ljubljana, Slovenia, pp 602-610. https://doi.org/10.3311/CCC2018-080

[21] Koskela, L. (2000) An exploration towards a production theory and its application to construction, ISBN 951-38-5565-1, VTTPublications, Kivimiehentie, Finland.

[22] Koskela, L.; Ballard, G.; Howell,G; Tommelein, I. (2002) The foundations of lean construction, Design and Construction: Building in Value https://doi.org/10.4324/9780080491080-23

[23] Winch,G. (2006) Towards a theory of construction as production by projects In: Building Research \& Information (2006) 34(2), 164174, https://doi.org/10.1080/09613210500491472

[24] Eber, W. Potentials of Artificial Intelligence in Construction Management, OTMC Organization, Technology and Management in Construction: an International Journal, DeGruyter and The University of Zagreb, Faculty of Civil Engineering, https://doi.org/10.2478/otmcj-2020-0002, Zagreb, 2020. 
[25] Shannon C. E. (1948): A Mathematical Theory of Communication. ISSN 0005-8580, In: Bell System Technical Journal. Short Hills N.J. 27.1948, (July, October), S. 379-423, 623-656. https://doi.org/10.1002/j.1538-7305.1948.tb00917.x

[26] Wassermann, S. Faust, K. (1994) Social Network Analysis. ISBN-10: 0521387078, Cambridge University Press, Cambridge.

[27] White, Douglas R., Jason Owen-Smith, James Moody, and Walter W. Powell. (2004). Networks, Fields and Organizations, , Computational and Mathematical Organization Theory 10:95-117 https://doi.org/10.1023/B:CMOT.0000032581.34436.7b

[28] Caldarelli, G; Vespignani, A (2007), Complex Systems and Interdisciplinary Science. Large Scale structure and dynamics of complex networks, World Scientific Publishing Co. Pte. Ltd. Vol.2., pp. 5-16. https://doi.org/10.1142/6455

[29] Ebeling, W.; Freund, J.; Schweitzer, F. (1998) Komplexe Strukturen: Entropie und Information, ISBN 9783815430323, Teubner Verlag, Stuttgart, https://doi.org/10.1007/978-3-322-85167-3,

[30] Eber, W., Managing Construction Projects: Developing Complexity into Complicatedness, Sustainable Built Environment D-A-CH Conference 2019, Graz University of Technology, Graz, Austria, 2019, https://doi.org/10.1088/1755-1315/323/1/012097

[31] Newman, M.E.J. (2003), The Structure and Function of Complex Networks, SIAM Review 45, pp. 167-256 https://doi.org/10.1137/S003614450342480

[32] Liening, A. (2017). Komplexität und Entrepreneurship, Springer Gabler, ISBN 978-3-658-13173-9, pp186ff, $431 \mathrm{ff}$.

[33] Strogatz, S. H. (2001), Exploring complex networks, Nature 410, p.268, https://doi.org/10.1038/35065725

[34] Hoffmann, W.; Körkemeyer, K. (2018) Zum Umgang mit der Komplexität von Bauvorhaben - Ergebnisse einer Expertenbefragung Bauingenieur 93 Springer, VDI-Verlag, Düsseldorf.

[35] Zadeh, L.A. (1965) Fuzzy sets in: Information and Control, Volume 8, Issue 3, June 1965, Elsevier, Amsterdam, pp 338-353 https://doi.org/10.1016/S0019-9958(65)90241-X 